\title{
$k$-TO-1 FUNCTIONS ON ARCS FOR $k$ EVEN
}

\author{
JO W. HEATH
}

\begin{abstract}
For exactly $k$-to- 1 functions from $[0,1]$ into $[0,1]$ :
(1) at least one discontinuity is required (Harrold),

(2) if $k=2$, then infinitely many discontinuities are needed, for any Hausdorff image space (Heath),

(3) if $k=4$, or if $k$ is odd, then there is such a function with only one discontinuity (Katsuura and Kellum),

and, it is shown here that

(4) if $k$ is even and $k>4$, then there is such a function with only two discontinuities, and no such function exists with fewer discontinuities.
\end{abstract}

I. Introduction. A function is $k$-to-1 if each point inverse has exactly $k$ elements, and it is at most $k$-to- 1 if each point inverse has at most $k$ elements.

Over 45 years ago, O. G. Harrold, Jr. proved in [2] that there is no continuous $k$-to-1 map from $[0,1]$ into $[0,1]$. In a recent paper [4], H. Katsuura and K. Kellum demonstrate that for each odd positive integer $k$, and for $k=4$, there is a $k$-to-1 function from $[0,1]$ into $[0,1]$ with exactly one discontinuity. They ask what is the minimum number of discontinuities for $k$-to- 1 functions from $[0,1]$ into $[0,1]$ with $k$ even. In [3] the author showed that any 2-to-1 function from [0,1] to a Hausdorff space requires infinitely many discontinuities. Thus only even numbers greater than 4 need be considered. Theorems 1 and 2 answer the question raised by Katsuura and Kellum for even integers at least 6:

THEOREM 1. If $f:[0,1] \rightarrow[0,1]$ is a $k$-to-1 function and $k$ is an even integer with $k>4$, then $f$ has at least two discontinuities.

THEOREM 2. If $k$ is an even integer greater than 4 then there is a $k$-to-1 function from $[0,1]$ into $[0,1]$ with only two discontinuities.

\section{Proof of Theorem 1.}

Lemma 1. Suppose $f:(0,1) \rightarrow(0,1)$ is a continuous map at most $k$-to-1, $p$ is in $(0,1)$, and $d$ is a positive number. Then there is a number $x$ with $x<p$ and $|x-p|<d$ such that:

1. either $f((x, p)) \subseteq(f(x), f(p))$, or $f((x, p)) \subseteq(f(p), f(x))$, and

Received by the editors July $21,1986$.

1980 Mathematics Subject Classification (1985 Revision). Primary 54C10, 26A15, 26 A03.

Key words and phrases. $k$-to-1 function, $k$-to-1 map.

(C1987 American Mathematical Society $0002-9939 / 87 \$ 1.00+\$ .25$ per page 
2. if $I$ is any subinterval of $(0,1)$ between $f(x)$ and $f(p)$, then there is a subinterval $J$ of $I$ such that every horizontal line $\{y=c\}$ with $c$ in $J$ intersects the graph of $f$ between $x$ and $p$ an odd number of times.

Proof. Since $f^{-1}(f(p))$ is finite, there is a positive number $d^{\prime}<d$ such that no point within $d^{\prime}$ of $p$ maps to $f(p)$ except $p$. Choose any number $x^{\prime}$ less than $p$ so that $\left|x^{\prime}-p\right|<d^{\prime}$. The set $f^{-1}\left(f\left(x^{\prime}\right)\right)$ is finite so there is an $x$ with $x^{\prime} \leqslant x<p$ and $f(x)=f\left(x^{\prime}\right)$ such that no point of $(x, p)$ maps to $f\left(x^{\prime}\right)$. Part 1 is true for this $x$. Note that the part 1 property implies that each point in $(0,1)$ is either a crossing point, a local maximum, or a local minimum for the graph of $f$.

Now suppose part 2 is false and suppose $f(x)<f(p)$. Then there is an interval $I$ in $(f(x), f(p))$ such that every subinterval contains a number $c$ where the line $\{y=c\}$ intersects the graph of $f$ between $x$ and $p$ an even number of times. Let $c_{1}$ be such a number in $I$ and let $n_{1}$ be the even number of times $\left\{y=c_{1}\right\}$ intersects the graph between $x$ and $p$. Since the graph goes continuously from $(x, f(x))$ below $\left\{y=c_{1}\right\}$ to $(p, f(p))$ above, an odd number of these intersection points must be crossings, say $j$ of them. Of the others, $i$ are local minima and $m$ are local maxima. Since $m+i$ is odd, one is larger, say $m>i$. Part 1 is true for each point in $f^{-1}\left(c_{1}\right)$ so there is an interval $I_{2}=\left(t, c_{1}\right)$ in $I$ small enough that if $c$ is in $I_{2}$ then $\{y=c\}$ intersects the graph between $x$ and $p$ at least $j+2 m$ times. From the negation of part 2 there is a number $c_{2}$ in $I_{2}$ such that the cardinality of this intersection is even, say $n_{2}$. Then

$$
n_{2}>j+2 m>j+m+i=n_{1} .
$$

This process can be continued until $n_{a}>k$. Since the line $\left\{y=c_{a}\right\}$ cannot intersect the graph of an at most $k$-to- 1 map more than $k$ times this is a contradiction.

Note 1 . Obviously the same properties hold to the right of $p$.

Note 2. Part 1 is a strengthening of a lemma found in Katsuura and Kellum [4].

Now, to prove Theorem 1 , suppose that $f:[0,1] \rightarrow[0,1]$ is a $k$-to- 1 function, with $k$ an even integer greater than 4 , and $f$ has only one discontinuity, $q$.

Claim 1. Without loss of generality it can be assumed that $f([0,1])$ is connected.

If $q=0$, then, since $f((0,1])$ is connected and $f(q)=f(x)$ for some $x$ in $(0,1]$, the image of $f$ is connected. If $0<q<1$, the connected sets $f([0, q))$ and $f((q, 1])$ are disjoint, and $f(q)=f(x)$ for some $x$ less than $q$, then $f$ restricted to $[0, q]$ is also a $k$-to-1 function with one discontinuity (Harrold proved there is at least one discontinuity) from $[0, q]$ to $[0,1]$.

Claim 2. Without loss of generality it can be assumed that $f([0,1])=(0,1)$.

Since the image is connected it is an interval with or without endpoints. Suppose 0 is in the image of $f$. One of 0 or 1 is not $q$, say $1 \neq q$. Let $z=1$ if $f(1)=0$ and $z=0$ if $f(1) \neq 0$. There are $k$ points that map to $0 ; n$ of them are neither 0,1 , nor $q$, with $n>2$ since $k>5$. Then $\left|f^{-1}(0)\right|=k \leqslant n+z+2$, where the 2 allows the possibility that $q$ and 0 are different and both map to 0 . Each of the $n$ points are local minima for $f$. Since $f$ restricted to each of $(0, q)$ and $(q, 1)$ is continuous and at most $k$-to-1, Lemma 1 holds. From part 1 of the lemma used at points of $f^{-1}(0)$ 
there is a number $e$ close to 0 so that the line $\{y=e\}$ intersects the graph of $f$ at least $2 n+z$ times. Since it intersects the graph exactly $k$ times the following is true:

$$
n+z+2 \geqslant k \geqslant 2 n+z
$$

from which it follows that $2>n$, a contradiction. Therefore 0 is not in the image of $f$.

Claim 3. The discontinuity $q$ is in $(0,1)$ and the limit of the images of any sequence converging to $q$ from the left is 0 and from the right is 1 (or the other way around). It was proved in Katsuura and Kellum [4] that each limit exists and is either 0 or 1 . If both limits were 1 , say, then there would be an interval of numbers $(0, e)$ not mapped onto, contradicting the fact that the image of $f$ is $(0,1)$. For the same reason, since both 0 and 1 limits must be achieved, $q$ must be interior to $[0,1]$ to have two sides in the domain.

Claim 4. $f(0)=f(1)$. If not, one of them, say $f(1)$, is not equal to $f(q)$. From Lemma 1 there are disjoint intervals $\left(a_{i}, b_{i}\right), i=1,2, \ldots, k-1$, about the points of $f^{-1}(f(1))$ other than 1 and an interval $\left(a_{k}, 1\right]$ disjoint from the others, that satisfy part 1.

Since points near $q$ map to values near 0 or 1 , there is a positive number $e$ so that the $e$-neighborhood about the line $\{y=f(1)\}$ contains no point of the graph of $f$ whose first coordinate lies outside the intervals $\left(a_{i}, b_{i}\right)$ and $\left(a_{k}, 1\right)$.

From part 2 of the lemma there are numbers $c$ in $(f(1), f(1)+e)$ and $c^{\prime}$ in $(f(1)-e, f(1))$ so that if $x_{i}$ is the point of $f^{-1}(f(1))$ in $\left(a_{i}, b_{i}\right)$ then

(1) if $x_{i}$ is a crossing point for the graph of $f$ on the line $\{y=f(1)\}$, then both of the lines $\{y=c\}$ and $\left\{y=c^{\prime}\right\}$ intersect the graph of $f$ an odd number of times between $a_{i}$ and $b_{i}$,

(2) if $x_{i}$ is a local minimum (or maximum) for $f$ then the line $\{y=c\}$ (or $\left.\left\{y=c^{\prime}\right\}\right)$ intersects the graph of $f$ an even number of times between $a_{i}$ and $b_{i}$ and the other line $\left\{y=c^{\prime}\right\}$ (or $\{y=c\}$ ) does not intersect the graph between $a_{i}$ and $b_{i}$, and

(3) one of the lines $\{y=c\}$ and $\left\{y=c^{\prime}\right\}$ intersects the graph an odd number of times between $a_{k}$ and 1 and the other not at all. It will be assumed here that $\{y=c\}$ is the one that intersects the graph over $\left(a_{k}, 1\right]$.

Let $m, n$, and $j$ denote the number of local maxima, local minima, and crossing points in $f^{-1}(f(1))-\{1\}$, respectively. Then

$\left|f^{-1}(c)\right|=k$ is the sum of $n$ even numbers plus $j+1$ odd numbers, and

$\left|f^{-1}(c)\right|=k$ is the sum of $m$ even numbers and $j$ odd numbers.

Since $k$ cannot be both even and odd there is a contradiction.

Claim 5. If Claim 3 is as stated and not the other way around, then $f([0, q)) \subseteq$ $(0, f(0)]$, and $f((q, 1]) \subseteq[f(1), 1)$.

Suppose there is a point $(a, b)$ on the graph of $f$ with $0<a<q, f(0)<b$, and $b$ is the largest such value. The inverse of $b$ contains $m$, say, local maxima points, and the number of crossing points, $j$, must be odd since they are all greater than $q$ and the graph of $f$ from $q$ to 1 goes from $y$-coordinates arbitrarily close to 1 near $q$ down to $f(1)$ at 1 , and $f(1)<b$. As in Claim 4 , there is a number $c^{\prime}<b, c^{\prime} \neq f(q)$, 
close enough to $b$ that $\left|f^{-1}\left(c^{\prime}\right)\right|=k$ is the sum of $m$ even numbers and $j$ odd numbers, which is impossible for the even number $k$.

Claim 6. There is a contradiction.

With the graph of $f$ to the left of $q$ below and on $\{y=f(0)\}$ and the graph to the right of $q$ above and on the same line, all of the points ( $m$ of them) of $f^{-1}(f(0))$ in $(0, q)$ are local maxima. Again, choose $c^{\prime}$ near $f(0)$ with $c^{\prime}<f(0), c^{\prime} \neq f(q)$, and the line $\left\{y=c^{\prime}\right\}$ intersects the graph in $k$ points, the sum of an odd number, corresponding to the point $(0, f(0))$, plus $m$ even numbers; a contradiction.

III. Proof of Theorem 2. The construction here is a generalization of an example in [4] which in turn used examples from [1] and [2]. The basic pieces of the graph of the function to be constructed are generalized $W$ 's and $M$ 's defined for a given integer $n$ :

(i) Choose $2 n+1$ distinct points in [0,1], $0=a_{0}<a_{1}<\cdots<a_{2 n}=b$, and define $W\left(a_{2 t}\right)=1$ and $W\left(a_{2 t+1}\right)=0$ for relevant $t$. Denote by $W(n)$ the piecewise linear extension to $[0,1]$.

(ii) Given an interval $[a, b]$, choose $2 n+2$ points $a=a_{0}<a_{1}<\cdots<a_{2 n+1}$ $=b$, and define $M\left(a_{2 t}\right)=a$ and $M\left(a_{2 t+1}\right)=b$ for relevant $t$. Let $M(a, b, n)$ denote the piecewise linear extension to a function from $[a, b]$ onto $[a, b]$. These basic pieces will be used to describe the basic units $B(n)$ and $A(t, n)$ for integers $t<n$ :

(iii) Define the function $B_{1}(n)$ from $[0,1]$ to $[0,1]$ by

$$
B_{1}(n)=(0,0)+\bigcup_{i=0}^{\infty} M\left(2^{-(i+1)}, 2^{-i}, n\right)
$$

Get the graph of $B_{2}(n)$ by first reflecting the graph of $B_{1}(n)$ about the vertical line $\{x=.5\}$ and then about the horizontal line $\{y=.5\}$.

(iv) Divide the rectangle $[0,3] \times[0,2]$ into six unit squares using matrix $S(i, j)$ notation. In the lower left square, $S(2,1)$, put the graph of $B_{1}(m)$; in $S(2,2)$ put the graph of $W(t)$; and in the upper right square, $S(1,3)$, put the graph of $B_{2}(n)$.

Consider the cardinality, $I(c)$, of the intersection of the line $\{y=c\}$ with this composite graph: If $1<c<2$ then $I(c)=2 n+1$. I(1) $=m+t+n+1$. If $0<c$ $<1$ then $I(c)=2 m+1+2 t$.

For the interior intersections to be constant, $2 n+1=m+t+n+1=2 m+1$ $+2 t$ is needed, i.e. $m+t=n$.

Given $n$ and $t$, then, set $m=n-t$ and denote the described function on [0,3] by $A_{1}(t, n)$. Denote by $A_{2}(t, n)$ the function constructed by reflecting the graph of $A_{1}(t, n)$ about the line $\{x=1.5\}$, and by $A_{3}(t, n)$ the function constructed by reflecting the graph of $A_{1}(t, n)$ about $\{y=1\}$.

Finally, since $k$ is an even integer greater than 4 it can be written as $(2 r+1)+$ $(2 s+1)$ where $r$ and $s$ are both positive. Define a continuous function $g$ from $[0,12]$ to $[0,4]$ by dividing $[0,12] \times[0,4]$ into eight $2 \times 3$ rectangles. Put $A_{1}(r-1, r)$ in the upper left rectangle $T(1,1), A_{2}(s-1, s)$ in $T(1,2), A_{3}(r-1, r)$ in $T(2,3)$, and $B_{1}(s)$ in the lower right rectangle $T(2,4)$. 
The wanted function $f$ will equal $g$ everywhere except at its two discontinuities, $f(3)=2$ and $f(9)=2$. The careful reader will agree that every point inverse has $k$ elements.

\section{REFERENCES}

1. P. Gilbert, n-to-one mappings of linear graphs, Duke Math. J. 9 (1942), 475-486.

2. O. G. Harrold, Jr., Exactly $(k, 1)$ transformations on connected linear graphs, Amer. J. Math. 62 (1940), 823-834.

3. J. W. Heath, Every exactly 2-to-1 function on the reals has an infinite number of discontinuities, Proc. Amer. Math. Soc. 98 (1986), 369-373.

4. H. Katsuura and K. Kellum, K-to-1 functions on an arc, preprint.

Department of Mathematics, Auburn University, Auburn, Alabama 36849 